January 2016

"Social insurance with competitive insurance markets and risk misperception"

Helmuth Cremer and Kerstin Roeder 


\section{Social insurance with competitive insurance markets and risk misperception $^{1}$}

\author{
Helmuth Cremer ${ }^{2}$ \\ Toulouse School of Economics
}

\author{
Kerstin Roeder ${ }^{3}$ \\ University of Augsburg
}

October 2015, revised January 2016

\footnotetext{
${ }^{1}$ Financial support from the Chaire "Marché des risques et creation de valeur" of the FdR/SCOR is gratefully acknowledged. This paper has been presented at the NTA conference in Boston; we thank all the participants and particularly our discussant, Louis Kaplow, for their comments and suggestions. We also thank Jean-Marie Lozachmeur his useful remarks.

${ }^{2}$ Corresponding author, Toulouse School of Economics, 21 Allée de Brienne, 31015 Toulouse, France. Email: helmuth.cremer@tse-fr.eu, Phone: +33 (0)5 61128606.

${ }^{3}$ University of Augsburg, Universitätsstr. 16, 86159 Augsburg, Germany, Email: kerstin.roeder@wiwi.uni-augsburg.de
} 


\begin{abstract}
This paper considers an economy where individuals differ in productivity and in risk. Rochet (1991) has shown that when private insurance markets offer full coverage at fair rates, social insurance is desirable if and only if risk and productivity are negatively correlated. This condition is usually shown to be satisfied for many health risks, but it appears to be violated for the old age dependency risk (mainly because longevity in turn is positively correlated with productivity). We examine the role of uniform and nonuniform social insurance to supplement a general income tax when neither public nor private insurers can observe individual risk and when it is positively correlated with wages. Consequently, a Rothschild and Stiglitz (1971) equilibrium emerges in the private insurance market and low-wage/low-risk individuals are not fully insured. We show that even when social insurance provided to the poor has a negative incentive effect, it also increases their otherwise insufficient insurance coverage. Social insurance to the rich produces exactly the opposite effects. Whichever of these effects dominates, some social insurance is always desirable. Finally, we introduce risk misperception which exacerbates the failure of private markets. The insurance term now reflects the combined failure brought about by adverse selection and misperception. Now the low-risk individuals are not only underinsured, but also pay a higher than fair rate. However, and rather surprisingly, it turns out that this does not necessarily strengthen the case for public insurance.
\end{abstract}




\section{Introduction}

A significant part of government intervention in the economy is, or can be, justified by redistributive considerations. A large variety of instruments is used, including taxation, transfers, price subsidies, in-kind transfers, pension benefits, and more generally social insurance. The set of these policies is often referred to as the "welfare state", a term which is used to characterize most industrialized countries, albeit to a different degree. From an economic perspective this raises many questions. For instance, which policies ought to be used or how should they be designed and financed?

A starting point for addressing these questions is the so-called Atkinson and Stiglitz (AS) theorem which states, roughly speaking, that when preferences are separable between labor supply and goods, any (incentive compatible) Pareto-efficient allocation can be implemented by using only a general income tax; see Atkinson and Stiglitz (1976) $1^{1}$ This theorem has far reaching implications. In particular, no commodity tax is needed, social insurance, in-kind transfers and public health insurance are not necessary, capital should not be taxed etc $2^{2}$ This result is explained by informational considerations. When the income tax is designed in an optimal way given the information that is available, an extra instrument is valuable only if it provides "better" information. In other words, if it improves the screening for variables which are private information and restrict the set of feasible policies. In the Mirrleesian world considered by AS, where individuals differ only in productivity, the separability of preferences implies that all these extra instruments do not provide any additional, pertinent information.

If we take the AS theorem at its face value, the welfare state should be downscaled dramatically and replaced by a "simple" well designed tax and (cash) transfer policy. It is by now well known that one of the major limitations of this result is that it relies on the assumption that individuals differ only in a single (non observable) dimension, namely productivity. When there are other factors of individual heterogeneity, including preferences or "risk", the result no longer applies $3^{3}$ While it is rather simple to show that many of the currently used instruments then have a potential role to play, the issue of the appropriate design of these policies is much more complicated.

\footnotetext{
${ }^{1}$ As shown by Laroque (2005) and Kaplow (2006) this result holds even when the income tax is not optimal. Roughly speaking, they show that starting from any tax scheme with income and commodity taxes, one can achieve a Pareto improvement by moving to a uniform commodity tax, while suitably reforming the income tax. Observe that this reform does not in general make the income tax optimal.

${ }^{2}$ See for instance Cremer (2003), Cremer and Gahvari (1997) and Cremer, Gahvari and Ladoux (1998).

${ }^{3}$ See Cremer et al. (1998) and Cremer, Pestieau and Rochet (2001).
} 
In this paper we focus on one of the both most prominent and most debated instrument, namely social insurance. Private insurance redistributes ex post, between states of nature. Premiums reflect individual risk. But only social insurance (or a suitable regulated private system) can effectively redistribute between ex ante heterogenous risk types. Put differently, social insurance can provide insurance against the "risk of being a bad risk", which private insurance cannot. This can be achieved through uniform premiums, or more general by a rate schedule where premium differences do not fully reflect risk differences.

Rochet (1991) and Cremer and Pestieau (1996) have shown that social insurance is desirable to supplement an optimal income tax, even when private insurance is actuarially fair, as long as productivity and risk are negatively correlated, i.e., when less productive individuals face the higher risk. This assumption is empirically supported for many health risks. These results thus support public health insurance contracts in which individual premiums are independent of their risk category. However, the assumption of a negative correlation is questionable for some risks, including the need for long-term care when dependency is due to cognitive affection like Alzheimer's disease. The incidence of cognitive disorders increases significantly with age, and longevity is positively correlated to productivity (as well as education and wealth); see Viscusi (1994), Gerdtham and Johannesson (2000), Cristia (2009). Not that higher income individuals are more likely per se to be affected by a cognitive disease, but lower income individuals are more likely to die of other courses before they reach the relevant age group. This longevity effect is also illustrated by the fact that $2 / 3$ of Alzheimer patients are women who have a higher life expectancy (National Academy on an Aging Society, 2000). For these kinds of risks a positive correlation between risk and productivity can no longer be ruled out 4 As shown by Cremer and Pestieau (1996), with fair private insurance markets, social insurance is then no longer desirable at least as long as the incentive constraint binds from high- to low-wage individuals 5 Intuitively, the willingness to pay for insurance is then higher for the high- than for the low-wage individuals and providing social insurance further reinforces an already binding incentive constraint.

Rochet's argument is quite powerful for the negative correlation case. When social insurance is desirable even with perfect private markets, it will certainly have a role to play when there are market failures in private markets. With positive correlation this reasoning does not apply

\footnotetext{
${ }^{4}$ See Cremer and Roeder (2013) for a detailed discussion of this issue. Observe that the epidemiologic literature shows that this longevity effect is mitigated by the fact that more educated individuals tend to have a larger "cognitive reserve" which tends to delay the onset of the cognitive impairment and shorten its duration (Reuser, Willekens and Bonneux, 2011).

${ }^{5}$ With a utilitarian social welfare function, this will be true unless the risk differential become so large that it outweighs the wage differential and the incentive constraint goes in the other direction.
} 
anymore and one can argue that social insurance does not come out as desirable, because private insurance is given an "unfair" advantage. More precisely the fair insurance assumption implies that private insurance has better information on individuals' risks than the government (or whatever administration is in charge of taxes and transfers). And the difference is drastic because private insurers perfectly observe risk, while public authorities do not observe it at all. This assumption is hard to defend.

In this paper we shall concentrate on the case of a positive correlation between productivity and risk and revisit the role of social insurance when neither public nor private insurers can observe the risk type. Instead, the private insurance market suffers from asymmetric information, and we assume that a Rothschild and Stiglitz (1971) equilibrium emerges. It is well-known that low-risk individuals will then be only partly insured. Consequently, we have a partial failure of the private insurance market. While public insurers do not have any superior knowledge of risk, they do have two advantages. First, they observe income levels and can implement a meanstested social insurance scheme. Second, they can make insurance mandatory. We examine whether this is sufficient to make a case for social insurance.

In a second step, we introduce the added feature that some individuals may misperceive their risk type and be overconfident. One would expect that this exacerbates the failure of the private markets and makes the role of public insurance even more compelling.

Methodologically this problem is quite challenging, even in an otherwise simple two-type setting. To keep it tractable we represent individuals' risk preferences by using Yaari's (1987) dual theory ${ }^{6}$ On the practical applied policy design side our model is meant to apply in particular to the dependency risk and the associated market for LTC insurance.7 As explained above, a positive correlation is likely to apply for old-age dependency. And myopia appears to be a pervasive phenomenon when it comes to severe forms of dependency; see Zhou-Richter, Browne and Grndl (2010), and Cremer and Roeder (2013) for a detailed discussion. Furthermore, in reality private insurance markets fail to a large extent to provide an appropriate coverage for this risk. Two of the major factors quoted to explain the thinness of private markets are precisely, adverse selection and myopia (risk misperception); see e.g., Brown and Finkelstein (2009).

We start by revisiting the fair insurance case within our dual theory setting; see Section 3 . While this mainly rediscovers Rochet's (1991) results, it constitutes an interesting benchmark for

\footnotetext{
${ }^{6}$ This allows us to derive a closed-form solution for the RS equilibrium.

${ }^{7}$ We do admittedly neglect some important features of LTC and particularly the role played by informal care, which currently represents a significant part of total care.
} 
the remainder of the analysis. Then, the introduction of adverse selection in the private market brings us to the RS setting; see Section 4. We consider both uniform and non-uniform (but self-selecting) social insurance. We show that in either case social insurance coverage provided to the low-wage (low-risk) individuals has two effects. First, an extra insurance effect because it increases coverage of otherwise underinsured individuals. Second, there is an incentive effect which is interpreted as in the fair private insurance case. We show that irrespective of the relative strength of these effects, some social insurance is always desirable. However, unless insurance is restricted in an ad hoc way to be uniform, only one of the types needs to receive social coverage. When the insurance effect outweighs the incentive effect (starting from an equilibrium without social insurance), the social insurance is designed for the low-wage individuals, who may or may not end up fully insured. Otherwise, and quite surprisingly, social insurance is targeted toward the high-wage individuals. Though exacerbating the insufficient coverage of the poor this policy relaxes an otherwise binding incentive constraint so that redistribution through the income tax is enhanced.

Finally, in Section 5 we introduce risk misperception, while continuing to consider an RS equilibrium in the private market. We consider a case where some high-risk individuals are overconfident and think they have a low risk. In equilibrium low-risk and overconfident individuals are pooled. Now, the low-risk individuals are not only underinsured, but also pay a higher than fair rate (effectively paying for the overconfident individuals who buy the same contract). Here we concentrate for technical reasons on uniform social insurance. Results are to some extent in line with expectations: there is an insurance term and incentive term. The insurance term now reflects the combined failure brought about by adverse selection and overconfidence. However, it also turns out that this does not necessarily strengthen the case for public insurance. Quite surprising, it turns out that overconfident individuals are of no direct relevance when it comes to the desirability of uniform social insurance. Overconfidence comes in indirectly though, because it increases the cost of private insurance which, in turn, affects the insurance term.

This paper builds on and relates to two strands of the literature on optimal (social) insurance. One theoretical literature that has considered optimal insurance and government redistribution problems jointly. In addition to Rochet (1991) and Cremer and Pestieau (1996) already mentioned, these include Chetty and Saez (2010) who like us have incomplete private insurance, but consider only one dimension of heterogeneity and restrict instruments to be linear. The closest predecessor to our paper is Nishimura (2009) who introduces adverse selection in the 
private market and the model used by Cremer and Pestieau (1996). However, they restrict their attention to uniform social insurance and do not consider misperception. They do show that the derivative with respect to the share of the risk covered by social insurance in the positive correlation case is ambiguous, with a negative incentive term (which they refer to a redistributive term) and a positive term which is similar to our additional insurance term 8 Second there are a few papers who study the effect of misperception in private markets and most notably Sandroni and Squintani (2007). While these authors look at some policies like mandatory insurance, they do not allow for income taxation.

\section{The model}

Individuals supply labor $\ell$ which comes with a (monetary) labor disutility $v(\ell)$. The ability to generate income differs among individuals, i.e., $w \in\left\{w_{r}, w_{p}\right\}$ with $0<w_{p}<w_{r}$. The fraction of low- and high-productivity individuals is $\nu_{p}$ and $\nu_{r}$ respectively. Individuals face a health risk; the monetary value of the potential loss is $L$. In addition to labor productivity, agents differ in their probability of incurring this loss $\pi \in\left\{\pi_{\ell}, \pi_{h}\right\}$ with $0<\pi_{\ell}<\pi_{h}<1$. Productivity and risk are perfectly correlated. In other words, each level of $w$ is associated with a unique level of $\pi$. So, we have either $\pi_{p} \equiv \pi_{\ell}>\pi_{r} \equiv \pi_{h}$ implying that the correlation between risk and productivity is negative or $\pi_{r} \equiv \pi_{\ell}>\pi_{p} \equiv \pi_{h}$ implying that it is positive. There is a private insurance market which offers insurance against this health risk. Additionally, a social insurance scheme which is financed by income taxation may exist.

We model individuals' risk preferences using Yaari's (1987) dual theory. Consider an individual with productivity $w$ incurring a damage $L$ with the probability $\pi$, and an insurance contract $(P, I)$, where $P$ is the premium, while $I$ is the level of coverage. Let $T \lessgtr 0$ denote the income tax and $D>0$ the social insurance benefits. This individual faces the lottery $X=(w \ell-v(\ell)-P-T, 1-\pi ; w \ell-v(\ell)-P-T-L+I+D, \pi)$. The utility associated with this lottery is given by

$$
\begin{aligned}
V(P, I ; w, \pi) & =(1-\phi(\pi))(w \ell-v(\ell)-T-P)+\phi(\pi)(w \ell-v(\ell)-T-P-L+I+D) \\
& =w \ell-v(\ell)-T-P+\phi(\pi)(-L+I+D),
\end{aligned}
$$

where $\phi(0)=0$ and $\phi(1)=1$. Risk aversion is represented by $\phi(\pi)>\pi$. Observe that the dual theory is based on the assumption that lotteries can be ranked, which here means that the

\footnotetext{
${ }^{8}$ See expression $\sqrt{27}$ below.
} 
individual is not better off when dependent then when in good health. In other words, (1) is only valid as long as $I+D \leq L$, that is as long as there is no overinsurance. Overinsurance cannot occur in equilibrium in our model. However, it can arise for some individual deviations. Specifically, we will show that mimicking individuals in the government incentive constraint may be overinsured. To deal with this in the simplest possible way, we assume that insurers will never pay out more than the effective loss $(L-D)$ to individuals. Formally, we rewrite $(1)$ as

$$
V(P, I ; w, \pi)=w \ell-v(\ell)-T-P+\min [\phi(\pi)(-L+I+D), 0],
$$

but to simplify notation we shall use the minimum operator only where necessary and stick to (1) otherwise $9^{9}$

The information structure is in line with Mirrleesian optimal tax models. We assume that gross income $y=w \ell$ is publicly observable and can be taxed according to a nonlinear function. Individual wages, $w$, labor supply, $\ell$ and loss probabilities $\pi$ are not publicly observable, nor are private insurance contracts $(P, I)$. However, the realization of an individual's risk is observable and social insurance benefits are paid only in that event. Income taxation is optimized, but the design of the tax schedule is not our main focus. Instead, we are interested in the desirability and design of social insurance given that income taxation is also optimized. To do so, we study the implied mechanism design problem where individuals are offered vectors $\Omega_{i}=\left(y_{i}, T_{i}, D_{i}\right)$ for $i=p, r$ specifying the before tax income, the income tax and the social insurance benefits. We study the optimal feasible (balanced budget) and incentive compatible mechanism. ${ }^{10}$

\subsection{Timing of the game}

Let us specify the timing of the mechanism design game. In a first stage, the government announces a mechanism which consists of two vectors $\Omega_{p}=\left(y_{p}, T_{p}, D_{p}\right)$ and $\Omega_{r}=\left(y_{r}, T_{r}, D_{r}\right)$. In the second stage, individuals ex ante (before the realization of the health risk) choose one of these vectors. Finally, in stage 3, individuals buy insurance coverage in the private market. The operation of this market depends on the information available to insurance companies. We first assume that they observe an individual's risk type. In a second step, we consider the

${ }^{9}$ If insurers would pay out the full claim in case of overinsurance, preferences would be represented by

$$
w \ell-v(\ell)-P-T-L+I+(1-\phi(1-\pi))(-L+I+D),
$$

given that being in good health is now the "bad" state of nature. Using this specification, rather than (2) would complicate the expressions, but not change the results. K: Are we sure of this?

${ }^{10}$ In practice this mechanism would have to be implemented by using tax cum social insurance schedules which are specified as functions of the observable variable $y$. We do not examine this problem because we are mainly interested in the levels of $D$ provided to the different types and this is part of the optimal mechanism. 
Rothschild-Stiglitz (RS) equilibrium of the private insurance market. Then, the insurers know that there are two types characterized by $\left(\Omega_{p}, \pi_{p}\right)$ and $\left(\Omega_{r}, \pi_{r}\right)$ respectively (both vectors being given at this point), but they do not observe who is who. In this scenario two premium-benefit contracts $\left(\pi_{p} I_{p}, I_{p}\right)$ and $\left(\pi_{r} I_{r}, I_{r}\right)$ are offered.

\section{$3 \quad$ Full information in the private insurance market}

When insurance companies observe an individual's risk type, they offer any insurance coverage at a fair price corresponding to each individual's health risk. Individuals choose their level of coverage to achieve full insurance, that is $I^{*}=L-D$; see Mossin's (1968) Theorem. With the considered information structure in the private market and the public sector, feasible allocations must satisfy the following incentive constraint

$$
\begin{aligned}
& y_{r}-v\left(\frac{y_{r}}{w_{r}}\right)-T_{r}-\pi_{r} I_{r}^{*}+\phi\left(\pi_{r}\right)\left(-L+I_{r}^{*}+D_{r}\right) \geq \\
& y_{p}-v\left(\frac{y_{p}}{w_{r}}\right)-T_{p}-\pi_{r} I_{r p}^{*}+\phi\left(\pi_{r}\right)\left(-L+I_{r p}^{*}+D_{p}\right) .
\end{aligned}
$$

That is the rich must be prevented from mimicking the poor. $I_{r p}^{*}$ thereby denotes the insurance coverage of the rich when they mimick the poor. Additionally, the resource constraint must hold. To incorporate redistributive concerns of the social planner, we study the optimal allocation that maximizes the sum of a strictly concave transformation of individual utilities $\Psi(V)$ with $\Psi^{\prime}(V)>0$ and $\Psi^{\prime \prime}(V)<0$. The government's problem then amounts to

$$
\begin{aligned}
\mathcal{L} & =\nu_{r} \Psi\left(y_{r}-v\left(\frac{y_{r}}{w_{r}}\right)-T_{r}-\pi_{r} I_{r}^{*}+\phi\left(\pi_{r}\right)\left(-L+I_{r}^{*}+D_{r}\right)\right) \\
& +\nu_{p} \Psi\left(y_{p}-v\left(\frac{y_{p}}{w_{p}}\right)-T_{p}-\pi_{p} I_{p}^{*}+\phi\left(\pi_{p}\right)\left(-L+I_{p}^{*}+D_{p}\right)\right) \\
& +\lambda\left(y_{r}-v\left(\frac{y_{r}}{w_{r}}\right)-T_{r}-\pi_{r} I_{r}^{*}+\phi\left(\pi_{r}\right)\left(-L+I_{r}^{*}+D_{r}\right)\right. \\
& \left.-y_{p}+v\left(\frac{y_{p}}{w_{r}}\right)+T_{p}+\pi_{r} I_{r p}^{*}-\phi\left(\pi_{r}\right)\left(-L+I_{r p}^{*}+D_{p}\right)\right)+\mu\left(\sum_{i} \nu_{i}\left(T_{i}-\pi_{i} D_{i}\right)\right),
\end{aligned}
$$

where $I_{r p}^{*}=I_{p}^{*}=L-D_{p}$, while $I_{r}^{*}=L-D_{r}$. The Lagrange multipliers with respect to the government's incentive constraint and the resource constraint are denoted by $\lambda$ and $\mu$ respectively. For an interior solution the FOCs for the rich are given by

$$
\begin{aligned}
\frac{\partial \mathcal{L}}{\partial D_{r}} & =\nu_{r} \Psi_{r}^{\prime}\left(-\pi_{r} \frac{\partial I_{r}^{*}}{\partial D_{r}}+\phi\left(\pi_{r}\right)\left(\frac{\partial I_{r}^{*}}{\partial D_{r}}+1\right)\right)+\lambda \pi_{r}-\mu \nu_{r} \pi_{r}=0 \\
\frac{\partial \mathcal{L}}{\partial T_{r}} & =-\nu_{r} \Psi_{r}^{\prime}-\lambda+\mu \nu_{r}=0 .
\end{aligned}
$$


Inserting (5) in (4) and using $I_{r}^{*}=L-D_{r}$ yields

$$
\frac{\partial \mathcal{L}}{\partial D_{r}}=\nu_{r} \Psi_{r}^{\prime} \pi_{r}+\lambda \pi_{r}-\nu_{r} \Psi_{r}^{\prime} \pi_{r}-\lambda \pi_{r} \equiv 0
$$

In words, irrespective of the correlation between income and risk, any level of social insurance for the rich, i.e., $D_{r} \in[0, L]$, yields the same level of welfare. Intuitively, $D_{r}$ does not allow to relax the incentive constraint because it is irrelevant for the mimicking individual. Since individuals are fully insured, $D_{r}$ is then essentially a redundant instrument as long as $T_{r}$ is used in an appropriate way.

Lets turn to the FOCs for the poor. Assuming an interior solution for $T_{p}$, we have

$$
\frac{\partial \mathcal{L}}{\partial T_{p}}=-\nu_{p} \Psi_{p}^{\prime}+\lambda+\mu \nu_{p}=0
$$

Differentiating with respect to $D_{p}$ and using $I_{r p}^{*}=I_{p}^{*}=L-D_{p}$ yields

$$
\frac{\partial \mathcal{L}}{\partial D_{p}}=\nu_{p} \Psi_{p}^{\prime} \pi_{p}-\lambda \pi_{r}-\mu \nu_{p} \pi_{p}=0
$$

Again inserting (7) in (8) amounts to

$$
\begin{aligned}
\frac{\partial \mathcal{L}}{\partial D_{p}} & =\nu_{p} \Psi_{p}^{\prime} \pi_{p}-\lambda \pi_{r}-\pi_{p} \nu_{p} \Psi_{p}^{\prime}-\lambda \pi_{p} \\
& =\lambda\left(\pi_{p}-\pi_{r}\right) \lessgtr 0 .
\end{aligned}
$$

When income and risk are positively correlated, i.e., $\pi_{p}<\pi_{r}$ then $D_{p}^{*}=0$. Otherwise, we have $D_{p}^{*}=L$. Note that when social insurance is restricted to be uniform that is $D_{p}=D_{r}=D$, then we have

$$
\frac{\partial \mathcal{L}}{\partial D}=\frac{\partial \mathcal{L}}{\partial D_{r}}+\frac{\partial \mathcal{L}}{\partial D_{p}}=0+\lambda\left(\pi_{p}-\pi_{r}\right)
$$

so that $D=L$ in case of a negative correlation and $D=0$ otherwise.

So far, we have been essentially confirming Rochet's results thereby showing that they continue to hold when individuals' risk preferences are represented using Yaari's (1987) dual theory. Summing up, equations (6), (9) and (10) imply that when all individuals have the same risk, the Atkinson and Stiglitz (1976) theorem applies and social insurance is not a useful instrument. Full social insurance is desirable if and only if loss probabilities differ across individuals and are negatively correlated. In that case, social insurance provides a larger utility gain to poor individuals than to (mimicking) rich. Observe that private insurance is then completely crowded out by social insurance. On the other hand, when there is a positive correlation, social insurance has an adverse effect on the incentive constraint. Intuitively, this means that it redistributes in 
the wrong direction, namely from the poor low-risk individuals to the rich high-risk ones. The following proposition summarizes the main results of this section.

Proposition 1 When insurance companies offer coverage at an actuarial fair rate, then

(i) full social insurance coverage for everyone is optimal when risk and productivity are negatively correlated,

(ii) no social insurance is desirable when risk and productivity are positively correlated.

While these results are interesting, they rely on the rather restrictive assumption that private insurers have better information about individuals' types than the tax or social insurance administration. This is at best debatable, and it would be more consistent to assume that private insurers and the administration have the same information concerning individuals characteristics. This is precisely what we'll do in the remainder of the paper. It is of course plain that the result for the negative correlation case won't change. If social insurance is beneficial when private markets are fair it will certainly continue to be beneficial when private markets are imperfect. Formally, one can see this by noting that the optimal solution under fair markets remains available when private markets are also affected by adverse selection. Since full social insurance crowds out these markets anyway, private market imperfections become irrelevant and we return to the fair markets case.

The interesting question arises for the case of positive correlation on which we focus in the remainder of the paper. Specifically, one may expect that the imperfections of private markets strengthen the case for social insurance and imply that (at least some) of it is desirable even under positive correlation.

\section{Adverse Selection in the private insurance market}

Assume from now on that risk and productivity are positively correlated so that $\pi_{r} \equiv \pi_{h}>\pi_{p} \equiv$ $\pi_{\ell}$. To solve our problem we proceed by backward induction and start by characterizing the last stage, namely the private insurance market RS equilibrium induced by the tax and social insurance policy. We assume throughout our analysis that a RS equilibrium exists.

\subsection{Private market equilibrium}

Insurers offer two contracts. One designed for the high risks with full insurance at a fair rate $\left(\pi_{r} I_{r}^{*}, I_{r}^{*}\right)$, where $I_{r}^{*}=L-D_{r}$. And one designed for low risks who also pay a fair price, but 
who may receive only partial insurance. Formally, $I_{p}^{*}$ is the largest level of $I_{p}$ which satisfies the incentive constraint on the private insurance market

$$
\begin{aligned}
& y_{r}-v\left(\frac{y_{r}}{w_{r}}\right)-T_{r}-\pi_{r} I_{r}+\phi\left(\pi_{r}\right)\left(-L+I_{r}+D_{r}\right) \geq \\
& y_{r}-v\left(\frac{y_{r}}{w_{r}}\right)-T_{r}-\pi_{p} I_{p}+\phi\left(\pi_{r}\right)\left(-L+I_{p}+D_{r}\right)
\end{aligned}
$$

and the condition $I_{p} \leq L-D_{p}$. Remember that at the second stage the rich receive full insurance, i.e., $I_{r}^{*}=L-D_{r}$. The solution to (11) when it is binding is given by

$$
I_{p}=\frac{\phi\left(\pi_{r}\right)-\pi_{r}}{\phi\left(\pi_{r}\right)-\pi_{p}}\left(L-D_{r}\right)
$$

which defines the insurance coverage for the poor $I_{p}^{*}$ as long as it $I_{p} \leq L-D_{p}$; otherwise we have $I_{p}^{*}=L-D_{p}$. Formally, $I_{p}^{*}$ is defined by

$$
I_{p}^{*}=\min \left[\frac{\phi\left(\pi_{r}\right)-\pi_{r}}{\phi\left(\pi_{r}\right)-\pi_{p}}\left(L-D_{r}\right), L-D_{p}\right] .
$$

Defining the maximum level of $D_{p}$ for which the incentive constraint in the private market is binding as

$$
\widetilde{D}_{p}=L-\frac{\phi\left(\pi_{r}\right)-\pi_{r}}{\phi\left(\pi_{r}\right)-\pi_{p}}\left(L-D_{r}\right)
$$

and noting that $\widetilde{D}_{p}>0$ for any $D_{r}$, because

$$
a \equiv \frac{\phi\left(\pi_{r}\right)-\pi_{r}}{\phi\left(\pi_{r}\right)-\pi_{p}}<1
$$

we can write (14) as

$$
\widetilde{D}_{p}=L(1-a)+a D_{r},
$$

which implies $\widetilde{D}_{p} \geq D_{r}$. When $D_{p}>\widetilde{D}_{p}$, the level of $I_{p}$ that yields full insurance (for the low-risk type) is sufficiently small not to be attractive to the high-risk type and equation (11) is not binding.

Having characterized the insurance market equilibrium, we can now turn to the determination of the optimal tax and social insurance policy. Formally, we have to determine the "best" consumption bundles $\Omega_{i}=\left(y_{i}, T_{i}, D_{i}\right)$ for $i=p, r$ which satisfy the resource constraint and are incentive compatible so that all individuals are at least as well off by choosing the bundle designed for them rather than by mimicking the other individuals. As usual we assume that only the downward constraint from $r$ to $p$ is binding. Before stating this problem we must have a closer look at this incentive constraint. The existence of private markets along with the 
assumption that private contracts are not publicly observable brings about one extra difficulty. The RS equilibrium is by definition incentive compatible so that in equilibrium all individuals will choose the bundle $\Omega_{i}=\left(y_{i}, T_{i}, D_{i}\right)$ and the contract $\left(\pi_{i} I_{i}, I_{i}\right)$ designed for them. However, this does not tell us which private contract an individual $r$ mimicking one of type $p$ will choose. This is the question we examine in the next subsection.

\subsection{Incentive constraint of the government}

Assume that the incentive constraint in the private market is binding. The mimicking individual chooses $I_{r}^{*}$ if (and only if)

$$
\begin{aligned}
& y_{p}-v\left(\frac{y_{p}}{w_{r}}\right)-T_{p}-\pi_{r} I_{r}^{*}+\min \left[\phi\left(\pi_{r}\right)\left(-L+I_{r}^{*}+D_{p}\right), 0\right] \geq \\
& y_{p}-v\left(\frac{y_{p}}{w_{r}}\right)-T_{p}-\pi_{p} I_{p}^{*}+\min \left[\phi\left(\pi_{r}\right)\left(-L+I_{p}^{*}+D_{p}\right), 0\right],
\end{aligned}
$$

which after simplification is implied by the insurance market incentive constraint (see equation 11) as long as $D_{p} \leq D_{r}$, so that he is not overinsured in any of the cases. Observe that in that case the mimicking type- $r$ individual is effectively indifferent between the two contracts $\left(\pi_{r} I_{r}^{*}, I_{r}^{*}\right)$ and $\left(\pi_{p} I_{p}^{*}, I_{p}^{*}\right)$.

Assume now that $D_{p}>D_{r}$. Then, the last term on the LHS of 16 is equal to zero since $I_{r}^{*}=L-D_{r}$ while the last term on the RHS is still negative (if the private market incentive constraint is binding). Consequently, the condition can be rewritten as

$$
-\pi_{r} I_{r}^{*} \geq-\pi_{p} I_{p}^{*}+\phi\left(\pi_{r}\right)\left(-L+I_{p}^{*}+D_{p}\right)
$$

while the incentive constraint in the private market can be written as

$$
-\pi_{r} I_{r}^{*}=-\pi_{p} I_{p}^{*}+\phi\left(\pi_{r}\right)\left(-L+I_{p}^{*}+D_{r}\right) .
$$

Combining these two expressions yields

$$
-\pi_{r} I_{r}^{*}=-\pi_{p} I_{p}^{*}+\phi\left(\pi_{r}\right)\left(-L+I_{p}^{*}+D_{r}\right) .
$$

That is, when $D_{p}>D_{r}$ condition $(16)$ is not satisfied and the mimicker prefers $I_{p}^{*}$ instead of $I_{r}^{*}$. while he is indifferent between the two contracts when $D_{p} \leq D_{r}$. We can thus write the government's problem as if the mimicking individuals would always choose $I_{p}^{*}$. The statement of the problem is then valid for both cases. This is true as long as the incentive constraint in the private market is binding. If not, that is when $D_{p}>\widetilde{D}_{p}$ is sufficiently large, we can no longer rule out the case where the mimicker chooses his "own" contract $\left(\pi_{r} I_{r}^{*}, I_{r}^{*}\right)$. We neglect this for the time being but reintroduce it when it will be relevant. 


\subsection{Optimal policy: problem and first-order conditions}

We are now in a position to state the problem determining the optimal allocation. The Lagrangean associated with this problem is

$$
\begin{aligned}
\mathcal{L} & =\nu_{r} \Psi\left(y_{r}-v\left(\frac{y_{r}}{w_{r}}\right)-T_{r}-\pi_{r} I_{r}^{*}+\phi\left(\pi_{r}\right)\left(-L+I_{r}^{*}+D_{r}\right)\right) \\
& +\nu_{p} \Psi\left(y_{p}-v\left(\frac{y_{p}}{w_{p}}\right)-T_{p}-\pi_{p} I_{p}^{*}+\phi\left(\pi_{p}\right)\left(-L+I_{p}^{*}+D_{p}\right)\right) \\
& +\lambda\left(y_{r}-v\left(\frac{y_{r}}{w_{r}}\right)-T_{r}-\pi_{r} I_{r}^{*}+\phi\left(\pi_{r}\right)\left(-L+I_{r}^{*}+D_{r}\right)\right. \\
& \left.-y_{p}+v\left(\frac{y_{p}}{w_{r}}\right)+T_{p}+\pi_{p} I_{p}^{*}-\phi\left(\pi_{r}\right)\left(-L+I_{p}^{*}+D_{p}\right)\right)+\mu\left(\sum_{i} \nu_{i}\left(T_{i}-\pi_{i} D_{i}\right)\right)
\end{aligned}
$$

Differentiating with respect to $D_{r}$ and $T_{r}$, and assuming that there is an interior solution for $T_{r}$ yields

$$
\begin{aligned}
\frac{\partial \mathcal{L}}{\partial D_{r}}= & \nu_{r} \Psi_{r}^{\prime}\left(-\pi_{r} \frac{\partial I_{r}^{*}}{\partial D_{r}}+\phi\left(\pi_{r}\right)\left(\frac{\partial I_{r}^{*}}{\partial D_{r}}+1\right)\right)+\nu_{p} \Psi_{p}^{\prime}\left(-\pi_{p}+\phi\left(\pi_{p}\right)\right) \frac{\partial I_{p}^{*}}{\partial D_{r}} \\
& -\lambda\left(\phi\left(\pi_{r}\right)-\pi_{p}\right) \frac{\partial I_{p}^{*}}{\partial D_{r}}-\lambda \pi_{r}-\mu \nu_{r} \pi_{r} \\
\frac{\partial \mathcal{L}}{\partial T_{r}}= & -\nu_{r} \Psi_{r}^{\prime}-\lambda+\mu \nu_{r}=0 .
\end{aligned}
$$

combining these two conditions and using 12 yields

$$
\begin{aligned}
\frac{\partial \mathcal{L}}{\partial D_{r}} & =\pi_{r} \nu_{r} \Psi_{r}^{\prime}+\left[\nu_{p} \Psi_{p}^{\prime}\left(\phi\left(\pi_{p}\right)-\pi_{p}\right)-\lambda\left(\phi\left(\pi_{r}\right)-\pi_{p}\right)\right] \frac{\partial I_{p}^{*}}{\partial D_{r}}+\lambda \pi_{r}-\mu \nu_{r} \pi_{r} \\
& =\left[\nu_{p} \Psi_{p}^{\prime}\left(\phi\left(\pi_{p}\right)-\pi_{p}\right)-\lambda\left(\phi\left(\pi_{r}\right)-\pi_{p}\right)\right] \frac{\partial I_{p}^{*}}{\partial D_{r}}, \\
& =-a\left[\nu_{p} \Psi_{p}^{\prime}\left(\phi\left(\pi_{p}\right)-\pi_{p}\right)-\lambda\left(\phi\left(\pi_{r}\right)-\pi_{p}\right)\right] .
\end{aligned}
$$

Before commenting on this expression, let us derive its counterpart for the variables pertaining to individual $p$. Differentiating the Lagrangean expression with respect to $D_{p}$ and $T_{p}$ yields

$$
\begin{aligned}
\frac{\partial \mathcal{L}}{\partial D_{p}} & =\nu_{p} \Psi_{p}^{\prime} \phi\left(\pi_{p}\right)-\lambda \phi\left(\pi_{r}\right)-\mu \nu_{p} \pi_{p} \\
\frac{\partial \mathcal{L}}{\partial T_{p}} & =-\nu_{p} \Psi_{p}^{\prime}+\lambda+\mu \nu_{p}=0 .
\end{aligned}
$$

Combining these expressions, we have

$$
\frac{\partial \mathcal{L}}{\partial D_{p}}=\nu_{p} \Psi_{p}^{\prime}\left(\phi\left(\pi_{p}\right)-\pi_{p}\right)-\lambda\left(\phi\left(\pi_{r}\right)-\pi_{p}\right) .
$$

The first term in in this expression is positive and shows the benefit of providing more insurance to individual $p$; recall that these individuals are only partially insured on the private market. 
The second term measures the costs in terms of incentive; the benefit of $D_{p}$ to the mimicker (type $r$ individuals) is larger than to the mimicked individuals. Since both terms are of opposite sign the expression evaluated at zero is ambiguous. Whether or not we want $D_{p}>0$ depends on the relative magnitude of the insurance and incentive terms. Recall that with fair insurance markets, social insurance was never optimal because it has an adverse incentive effect (favoring the mimicking rich more than the poor). When there is asymmetric information in the private market, there continues to be a negative incentive effect. However, there is also a positive effect because it increases insurance coverage of the poor (who are less than fully insured in the RS equilibrium). Observe that

$$
\frac{\partial \mathcal{L}}{\partial D_{r}}=-a \frac{\partial \mathcal{L}}{\partial D_{p}} .
$$

Consequently, the interpretation of $(22)$ is similar to that of (25) except that the signs of the two effects are reversed.

Before proceeding with the characterization of $D_{p}$ and $D_{r}$ let us consider the case where we impose the ad hoc assumption that $D$ is required to be uniform so that $D_{p}=D_{r}=D$. We then have from $(26)$

$$
\frac{\partial \mathcal{L}}{\partial D}=\frac{\partial \mathcal{L}}{\partial D_{p}}+\frac{\partial \mathcal{L}}{\partial D_{r}}=\frac{\partial \mathcal{L}}{\partial D_{p}}(1-a)
$$

Substituting from (25) and rearranging successively yields

$$
\begin{aligned}
\frac{\partial \mathcal{L}}{\partial D} & =(1-a) \nu_{p} \Psi_{p}^{\prime}\left[\phi\left(\pi_{p}\right)-\pi_{p}\right]-\lambda\left[\phi\left(\pi_{r}\right)-\pi_{p}\right]+a \lambda\left[\phi\left(\pi_{r}\right)-\pi_{p}\right] \\
& =(1-a) \nu_{p} \Psi_{p}^{\prime}\left[\phi\left(\pi_{p}\right)-\pi_{p}\right]-\lambda\left[\pi_{r}-\pi_{p}\right] .
\end{aligned}
$$

Interestingly, the incentive term is now exactly the same as in the fair private market case. We again have an insurance term similar to that in 25 but mitigated by the concomitant increase in $D$ for the rich. Either way, we obtain that even simple uniform social insurance may be welfare improving due to private insurance market imperfections. And this is the case even though its incentive effect is negative.

Let us now return to the case where social insurance is not restricted to be uniform. Expressions 22 and 25 are valid as long as $I_{p}^{*}$ is given by 12 that is as long as $D_{p} \leq \widetilde{D}_{p}$; otherwise we have $\partial I_{p}^{*} / \partial D_{r}=0$ and $\partial I_{p}^{*} / \partial D_{p}=-1$ so that equation 22 reduces to

$$
\frac{\partial \mathcal{L}}{\partial D_{r}} \equiv 0
$$

while 25$)$ is replaced by

$$
\frac{\partial \mathcal{L}}{\partial D_{p}}=\nu_{p} \Psi_{p}^{\prime} \pi_{p}-\lambda \pi_{p}-\mu \nu_{p} \pi_{p}=-\pi_{p} \frac{\partial \mathcal{L}}{\partial T_{p}},
$$


which is equal to zero as long as there is an interior solution for $T_{p}$. When both individuals (as well as the mimicker in the incentive constraint) are fully insured, $D_{p}$ is equivalent to a transfer to individual $p$ (a negative tax) and the instrument is redundant. Consequently, nothing can be gained by setting $D_{p}>\widetilde{D}_{p}{ }^{11}$

\subsection{Optimal policy: no redistributive concerns}

The expressions presented in the previous subsection and their interpretations have shown that both redistributive and efficiency considerations are relevant. In other words, in addition to its redistributive role studied in Section 3, social insurance now corrects the partial insurance market failure brought about by adverse selection. Section 3 has shown that when there is no market failure so that redistribution is the only relevant concern, social insurance is not desirable under positive correlation. Before studying the general solution, let us consider the "opposite" case namely where the government does not care about redistribution, i.e. $\Psi^{\prime}(V)=1$, but when there is adverse selection in the private insurance market. Recall that without any government intervention, the low-risk agents have only partial insurance coverage $I_{p}^{*}<L$ in the RS equilibrium. This is, given that individuals are risk averse, i.e. $\phi\left(\pi_{i}\right)>\pi_{i}$, an inefficient allocation. Intuitively, one would expect that social insurance at the very least ought to complete the poor's coverage to achieve full insurance. To see how this is brought out by the FOCs observe first that absent of redistributive concern it is plain that the solution implies $\lambda=0$. Since taxes and transfers have no impact on social welfare, they can always be set so that the self-selection constraint is satisfied at no welfare cost. In other words, a first-best outcome can be implemented. For instance, one can set $D_{r}=0$ (the rich are fully insured anyway) and provide social insurance to the poor of $D_{p}=L-I_{p}^{*}$ "financed" by a tax of $\pi_{r} D_{p}$ levied on the poor. Then, both individuals are fully insured and the rich individuals' incentives to mimick are nil, since they would pay the same price for social insurance as they do for private insurance. And it is obvious that the poor do not want to mimick the rich for otherwise they would have to pay $\pi_{r} L$ to get full insurance. With this policy there is a budgetary surplus of $\nu_{p} D_{p}\left(\pi_{r}-\pi_{p}\right)$. It

\footnotetext{
${ }^{11}$ As mentioned above, when $D_{p}>\widetilde{D}_{p}$ and $D_{p}$ is sufficiently large, the mimicking individual may prefer to buy $I_{p}^{*}$ in the private market. In that case, 28$]$ and $[29$ are replaced by

$$
\begin{gathered}
\frac{\partial \mathcal{L}}{\partial D_{r}}=\lambda\left(\phi\left(\pi_{r}\right)-\pi_{p}\right)>0, \\
\frac{\partial \mathcal{L}}{\partial D_{p}}=-\lambda\left(\phi\left(\pi_{r}\right)-\pi_{p}\right)<0 .
\end{gathered}
$$

Consequently, we continue to find that nothing can be gained by setting $D_{p}>\widetilde{D}_{p}$.
} 
can be redistributed through a uniform transfer which has no impact on the incentive constraint yielding $T_{p}=\pi_{r} D_{p}-\nu_{p} D_{p}\left(\pi_{r}-\pi_{p}\right)$ and $T_{r}=-\nu_{p} D_{p}\left(\pi_{r}-\pi_{p}\right)$.

To show that this solution indeed satisfies the FOCs note that with $\lambda=0$ and $\Psi^{\prime}=1$, equations (21) and (24) yield $\mu=1$. It then follows from equations (25), (29) and (28) that $\partial \mathcal{L} / \partial D_{p}>0$ up to $D_{p}=L-I_{p}^{*}$ and equal to zero when $D_{p}=L-I_{p}^{*}$, while $\partial \mathcal{L} / \partial D_{p}=0$. Observe that this characterizes the minimal level of $D_{p}$ which implements a first-best solutions. Higher levels of social insurance leave welfare unaffected, but the same allocation could also be implemented in a "trivial" way by providing say uniform full social insurance $D=L$; see equation 27.

\subsection{Optimal policy: general solution}

We are now in a position to characterize the solution. To do this in the simplest possible way, the graphical illustration provided in Figure 1 is useful. The figure projects our problems into the

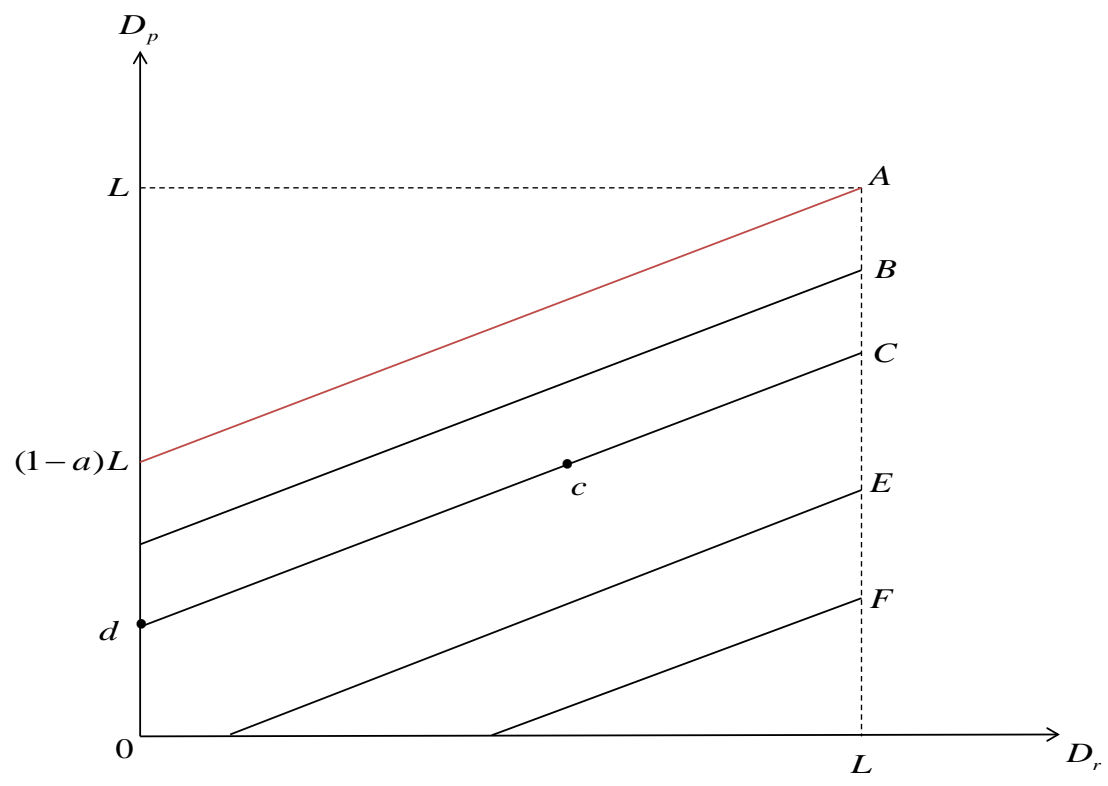

Figure 1: Welfare level curves in the $\left(D_{r}, D_{p}\right)$ space

$\left(D_{r}, D_{p}\right)$ space. Clearly, neither of these variables will exceed $L$ so we can restrict our attention to the domain $[0, L] \times[0, L]$. The lines $B, C, E, F$ represent some randomly selected level curves 
of $\mathcal{L}$. To represent them we use 26 , which implies that these are all straight lines with slope of a. In addition, $A$ represents equation 15 defining $\widetilde{D}_{p}$ as function of $D_{r}$. It is also a straight line, with slope $a$ and thus parallel to the level curves. We can neglect the area above $A$ because we already know from the previous subsection that nothing can be gained by setting $D_{p}>\widetilde{D}_{p}$. For instance, point $c$, with both insurance benefits strictly positive yields the same level of welfare as $d$, on the vertical axis so that $D_{r}=0$. Similarly, any point on $E$ can be duplicated by the intersection of this line with the horizontal axis.

This figure shows that one of the two instruments is always redundant. Nothing can be gained by choosing a point at the interior of the square. Assuming that redundant instruments are not used it is plain from the graphical representation that the solution is then given by $D_{r}=0$ and some $D_{p}^{*} \in[0,(1-a) L]$, when $\partial \mathcal{L}(0,0) / \partial D_{p}>0$ i.e., when 25 evaluated at $D_{r}=D_{p}=0$ is strictly positive. Note that from 26 this automatically implies that $\partial \mathcal{L}(0,0) / \partial D_{r}<0$. On the other hand, when $\partial \mathcal{L}(0,0) / \partial D_{r}>0$ so that $\partial \mathcal{L}(0,0) / \partial D_{p}<0$ we can set $D_{p}=0$ and determine the best $D_{r}^{*} \in[0, L]$.

Intuitively, when $\partial \mathcal{L}(0,0) / \partial D_{p}>0$, we know from the discussion in the previous subsection that the positive insurance effect of $D_{p}$ outweighs the negative incentive effect. The opposite is true for $D_{r}$. Consequently, we can set $D_{r}=0$ and the solution of $D_{p}$ is then either given by an "interior" solution in the interval $D_{p}^{*} \in[0,(1-a) L)$ such that 25$)$ is zero. Alternatively, if welfare continues to increase up to $(1-a) L$, we obtain $D_{p}^{*}=(1-a) L$, that is $D_{p}^{*}=\widetilde{D}_{p}$ and social insurance for $p$ is increased until the incentive constraint in the private market is no longer binding so that poor individuals are fully insured. This outcome is very much "as expected" but one has to keep in mind that it may or may not be the optimal policy.

The opposite case where $\partial \mathcal{L}(0,0) / \partial D_{p}<0$ is more surprising. We can then set $D_{p}=0$ and $0<D_{r}^{*} \leq L$. This means that while $p$ is already underinsured, while $r$ is fully insured anyway, it proves beneficial to provide social insurance to the rich. This insurance, in turn will decrease the private insurance available to the poor, via the private market incentive constraint. So, even though the policy is aimed at redistributing to $p$, we give social insurance to $r$, leaving $p$ underinsured. This is beneficial here because the incentive effect outweighs the insurance effect. In words, the policy relaxes the incentive constraint, which in turn makes it possible to redistribute more via income taxation.

Summing up, it turns out that unless $\partial \mathcal{L}(0,0) / \partial D_{p}=0$ which is not generically true, the solution always implies that some social insurance is provided to one type of individuals. This 
is in sharp contrast with the fair insurance market case where no social insurance was desirable under positive correlation.

Observe also that in either was the solution is obtained by balancing the positive effect of correcting a market failure with the adverse redistributive impact that social insurance has under positive correlation between risk and ability. In that sense the general solution is roughly speaking a "convex combination" of the outcomes achieved in the two extreme cases presented in Section 3 (no market failure) and in Subsection 4.4 (no redistribution).

The main results of this section are summarized in the following proposition.

Proposition 2 When the private insurance market is characterized by the Rothschild-Stiglitz equilibrium and the correlation between income and risk is positive, then

(i) When $\Psi^{\prime}=1$ so that there is no concern for redistribution, a first-best outcome with full insurance for all can be implemented. The minimal amount of social insurance to achieve this is given by $D_{p}=L-I_{p}^{*}$ and $D_{r}=0$. In other words, social insurance merely completes the market coverage provided to the poor.

(ii) When $\Psi^{\prime}$ is strictly convex so that redistribution matters, the solution is second-best and we have

(a) The rich are always fully insured (be it private or public) while the poor may or may not be fully insured.

(b) It is always desirable to provide some social insurance.

(c) Depending on the strength of the insurance and incentive effect, social insurance is always redundant for one type of individuals. It is positive for the poor if the insurance effect outweighs the incentive effect and it is positive for the rich otherwise.

\section{Misperception and social insurance}

We now introduce an additional source of imperfection in private insurance markets, namely the fact that some high-risk individuals may be overconfident and misleadingly think that they have a low health risk. As argued above this appears to be the case in reality, in particular for the dependency risk associated with long-term care needs. One can expect this to further strengthen the case for social insurance.

We assume that part of the high-risk individuals are overconfident concerning their health risk. Formally, we then have three types of individuals indexed by $r, o$ and $p$ and in (strictly positive) proportions $\nu_{r}, \nu_{o}$ and $\nu_{p}$. Types $r$ and $p$ are the same as before and we have $w_{r}>w_{p}$ 
and $\pi_{r} \equiv \pi_{h}>\pi_{p} \equiv \pi_{\ell}$. Type $o$ individuals are the same as type $r$ except that they are overconfident and think that their risk is $\pi_{\ell}$ while in reality it is $\pi_{h}$. And it is this perceived risk which determines their demand in the insurance market. Consequently, insurance companies cannot screen between overconfident and low-risk agents. That is, individuals are separated on the basis of their beliefs.

The insurance market equilibrium is then as follows ${ }^{12}$ For high-risk individuals the presence of overconfident individuals changes nothing; they get full insurance at an actuarial fair price. Low-risk agents, by contrast, get a different contract which insures self-selection. We assume that social insurance is uniform so that $D_{p}=D_{r}=D_{o}=D$. The average probability of a damage for overconfident and low-risk agents is given by

$$
\pi_{p o} \equiv \frac{\nu_{o} \pi_{r}+\nu_{p} \pi_{p}}{\nu_{o}+\nu_{p}}
$$

Specifically, the coverage, $I_{p o}$, low-risk and overconfident agents get is determined by the following self-selection constraint

$$
\begin{aligned}
& y_{r}-v\left(\frac{y_{r}}{w_{r}}\right)-T_{r}-\pi_{r} I_{r}+\phi\left(\pi_{r}\right)\left(-L+I_{r}+D\right) \geq \\
& y_{r}-v\left(\frac{y_{r}}{w_{r}}\right)-T_{r}-\pi_{p o} I_{p o}+\phi\left(\pi_{r}\right)\left(-L+I_{p o}+D\right),
\end{aligned}
$$

where $I_{r}^{*}=L-D_{r}$. Formally, $I_{p o}^{*}$ is then defined by

$$
I_{p o}^{*}=\frac{\phi\left(\pi_{r}\right)-\pi_{r}}{\phi\left(\pi_{r}\right)-\pi_{p o}}(L-D) .
$$

Note that $I_{p o}^{*}$ is the same for overconfident and low-risk individuals. In the following analysis it will be helpful to define

$$
b \equiv \frac{\phi\left(\pi_{r}\right)-\pi_{r}}{\phi\left(\pi_{r}\right)-\pi_{p o}}<0 .
$$

Turning to the incentive constraints in the governments problem, we consider the following three

\footnotetext{
${ }^{12}$ Existence may require some additional restriction, in particular on proportions which we neglect here for simplicity.
} 
constraints

$$
\begin{aligned}
& y_{r}-v\left(\frac{y_{r}}{w_{r}}\right)-T_{r}-\pi_{r} I_{r}^{*}+\phi\left(\pi_{r}\right)\left(-L+I_{r}^{*}+D\right) \geq \\
& y_{p}-v\left(\frac{y_{p}}{w_{r}}\right)-T_{p}+\pi_{r} I_{r}^{*}+\phi\left(\pi_{r}\right)\left(-L+I_{r}^{*}+D\right), \\
& y_{r}-v\left(\frac{y_{r}}{w_{r}}\right)-T_{r}-\pi_{r} I_{r}^{*}+\phi\left(\pi_{r}\right)\left(-L+I_{r}^{*}+D\right) \geq \\
& y_{o}+v\left(\frac{y_{o}}{w_{r}}\right)+T_{o}+\pi_{r} I_{r}^{*}+\phi\left(\pi_{r}\right)\left(-L+I_{r}^{*}+D\right), \\
& y_{o}-v\left(\frac{y_{o}}{w_{r}}\right)-T_{o}-\pi_{p o} I_{p o}^{*}+\phi\left(\pi_{p}\right)\left(-L+I_{p o}^{*}+D\right) \geq \\
& y_{p}-v\left(\frac{y_{p}}{w_{r}}\right)-T_{p}+\pi_{p o} I_{p o}^{*}+\phi\left(\pi_{p}\right)\left(-L+I_{p o}^{*}+D\right),
\end{aligned}
$$

The first of these constraints prevents $r$ from mimicking $p$, the second $r$ from mimicking $o$, and the third $o$ from mimicking $p$. Observe that these constraints assume that mimicking individuals choose their "own" contract in the private market. With uniform social insurance this is necessarily true and follows directly from 30 .

Combining the incentive constraints shows that (34) and (35) together imply (33). In words, when the constraints from $r$ to $o$ and from $o$ to $p$ are satisfied, the constraint from $r$ to $p$ is necessarily also satisfied. There is thus no need to impose (33) as separate constraint.

The Lagrangean of the government is then given by

$$
\begin{aligned}
\mathcal{L} & =\nu_{r} \Psi\left(y_{r}-v\left(\frac{y_{r}}{w_{r}}\right)-T_{r}-\pi_{r} I_{r}^{*}+\phi\left(\pi_{r}\right)\left(-L+I_{r}^{*}+D\right)\right) \\
& +\nu_{p} \Psi\left(y_{p}-v\left(\frac{y_{p}}{w_{p}}\right)-T_{p}-\pi_{p o} I_{p o}^{*}+\phi\left(\pi_{p}\right)\left(-L+I_{p o}^{*}+D\right)\right) \\
& +\nu_{o} \Psi\left(y_{o}-v\left(\frac{y_{o}}{w_{r}}\right)-T_{o}-\pi_{p o} I_{p o}^{*}+\phi\left(\pi_{r}\right)\left(-L+I_{p o}^{*}+D\right)\right) \\
& +\lambda_{1}\left(y_{r}-v\left(\frac{y_{r}}{w_{r}}\right)-T_{r}-\pi_{r} I_{r}^{*}+\phi\left(\pi_{r}\right)\left(-L+I_{r}^{*}+D\right)\right. \\
& \left.-y_{o}+v\left(\frac{y_{o}}{w_{r}}\right)+T_{o}+\pi_{r} I_{r}^{*}-\phi\left(\pi_{r}\right)\left(-L+I_{r}^{*}+D\right)\right) \\
& +\lambda_{2}\left(y_{o}-v\left(\frac{y_{o}}{w_{r}}\right)-T_{o}-\pi_{p o} I_{p o}^{*}+\phi\left(\pi_{p}\right)\left(-L+I_{p o}^{*}+D\right)\right. \\
& \left.-y_{p}+v\left(\frac{y_{p}}{w_{r}}\right)+T_{p}+\pi_{p o} I_{p o}^{*}-\phi\left(\pi_{p}\right)\left(-L+I_{p o}^{*}+D\right)\right)+\mu\left(\sum_{i} \nu_{i}\left(T_{i}-\pi_{i} D\right)\right)
\end{aligned}
$$


The FOCs are given by

$$
\begin{aligned}
\frac{\partial \mathcal{L}}{\partial D}= & \nu_{r} \Psi_{r}^{\prime} \pi_{r}+\nu_{p} \Psi_{p}^{\prime}\left[-\pi_{p o} \frac{\partial I_{p o}^{*}}{\partial D}+\phi\left(\pi_{p}\right)\left(1+\frac{\partial I_{p o}^{*}}{\partial D}\right)\right] \\
& +\nu_{o} \Psi_{o}^{\prime}\left[-\pi_{p o} \frac{\partial I_{p o}^{*}}{\partial D}+\phi\left(\pi_{r}\right)\left(1+\frac{\partial I_{p o}^{*}}{\partial D}\right)\right]-\mu\left(\nu_{r} \pi_{r}+\nu_{p} \pi_{p}+\nu_{0} \pi_{r}\right) \\
\frac{\partial \mathcal{L}}{\partial T_{r}}= & -\nu_{r} \Psi_{r}^{\prime}-\lambda_{1}+\mu \nu_{r}=0 \\
\frac{\partial \mathcal{L}}{\partial T_{p}}= & -\nu_{p} \Psi_{p}^{\prime}+\lambda_{2}+\mu \nu_{p}=0 \\
\frac{\partial \mathcal{L}}{\partial T_{o}}= & -\nu_{o} \Psi_{o}^{\prime}+\lambda_{1}-\lambda_{2}+\mu \nu_{o}=0
\end{aligned}
$$

Using (31) and (32), equation (37) can be rewritten as

$$
\begin{aligned}
\frac{\partial \mathcal{L}}{\partial D}= & \nu_{r} \Psi_{r}^{\prime} \pi_{r}+\nu_{p} \Psi_{p}^{\prime}\left[\pi_{p o} b+\phi\left(\pi_{p}\right)(1-b)\right]+\nu_{o} \Psi_{o}^{\prime}\left[\pi_{p o} b+\phi\left(\pi_{r}\right)(1-b)\right] \\
& -\mu\left(\nu_{r} \pi_{r}+\nu_{p} \pi_{p}+\nu_{0} \pi_{r}\right)
\end{aligned}
$$

observe that $\pi_{p o} b+\phi\left(\pi_{r}\right)(1-b)=b\left[\pi_{p o}-\phi\left(\pi_{r}\right)\right]+\phi\left(\pi_{r}\right)=\pi_{r}$. Substituting, we have

$$
\begin{aligned}
\frac{\partial \mathcal{L}}{\partial D}= & \nu_{r} \Psi_{r}^{\prime} \pi_{r}+\nu_{o} \Psi_{o}^{\prime} \pi_{r}+\nu_{p} \Psi_{p}^{\prime} \pi_{p}-\mu\left(\nu_{r} \pi_{r}\right. \\
& \left.+\nu_{p} \pi_{p}+\nu_{0} \pi_{r}\right)+\nu_{p} \Psi_{p}^{\prime}\left[\pi_{p o} b+\phi\left(\pi_{p}\right)(1-b)-\pi_{p}\right] \\
= & -\lambda_{1} \pi_{r}+\lambda_{2} \pi_{p}+\left(\lambda_{1}-\lambda_{2}\right) \pi_{r}+\nu_{p} \Psi_{p}^{\prime}\left[\pi_{p o} b+\phi\left(\pi_{p}\right)(1-b)-\pi_{p}\right] \\
= & \nu_{p} \Psi_{p}^{\prime}\left[\pi_{p o} b+\phi\left(\pi_{p}\right)(1-b)-\pi_{p}\right]-\lambda_{2}\left(\pi_{r}-\pi_{p}\right)
\end{aligned}
$$

Equation (42) includes ones again an insurance term and an incentive term. The incentive term is negative given $\pi_{r}>\pi_{p}$, and its interpretation is exactly the same as in Section 3 . When the mimicker has a higher risk than the mimicked individual, uniform social insurance reinforces the incentive constraint. Interestingly, only individuals $r$ and $p$ matter for this term; all effects pertaining to individuals $o$ cancel out. This is because they appear both as mimicked and mimicking individual in the incentive constraints.

Turning to the insurance term, it is positive because the first two terms in brackets represent a convex combination of two terms which are both larger than $\pi_{p}$. Recall that $b=\partial I_{p o}^{*} / \partial D$ is defined by $(32)$ and satisfies $0<b<1$. To interpret this term first observe that given the way we combined the FOCs, we are effectively considering a variation $\mathrm{d} D$ which is financed by an increase in $T_{i}$ so that $\mathrm{d} T_{i}=\pi_{i} \mathrm{~d} D$. In words, individuals face a tax increase which equals the expected cost of the extra social insurance they receive. The first term in 42 measures the impact of this variation on the contribution to social welfare of $p$. To see this note that the 
first term in brackets measures the premium savings in the private market. The second term measures the benefit of the extra insurance protection; it accounts for the fact that when $D$ increases by $1, I_{p o}^{*}$ decreases by $b$. Individuals thus receive a net extra benefit of $(1-b)$, which is weighted by $\phi\left(\pi_{p}\right)$ since it occurs in the bad state of nature. The third term represents the cost of the "compensating" tax increase. Finally, the term in brackets is multiplied by $\nu_{p} \Psi_{p}^{\prime}$ to convert individual utility into contributions to social welfare.

It may be surprising at first, that only the impact on $p$ appears in 42 . However, this is also easily understood. First, the counterpart of this term for $r$ obviously vanishes because these individuals are already fully insured. Consequently, public insurance only crowds out private coverage at the same cost. Turning to individuals $o$, the corresponding bracketed term would be $\left[\pi_{p o} b+\phi\left(\pi_{r}\right)(1-b)-\pi_{r}\right]$. We have already shown above that $\pi_{p o} b+\phi\left(\pi_{r}\right)(1-b)=\pi_{r}$ so that this expression also vanishes. Recall also that this equation is obtained by substituting for $b$, that is effectively by making use of the private market incentive constraint. Intuitively, this makes sense. Individuals $r$ and $o$ are alike as far as their real preferences are concerned and also with the considered variation $\mathrm{d} T_{r}=\mathrm{d} T_{0}=\pi_{r} \mathrm{~d} D$. Consequently, if $o$ would become better off by the considered variation, $r$ mimicking $o$ would be better off too and this would violate the IC constraint. In other words, the result arises because $I_{p o}^{*}$ adjusts to satisfy the private market IC constraint.

It thus turns out that individuals $o$ are of no direct relevance when it comes to the desirability of uniform social insurance. Overconfidence comes in indirectly though because it increases the cost of private insurance for $\pi_{p}$; they now pay insurance protection at a price of $\pi_{p o}>\pi_{p}$ which is larger than the fair rate (because they are pooled with individuals $o$ ).

To sum up, uniform social insurance is desirable if (and only if) the insurance term (evaluated at $D=0$ ) outweighs the incentive term. Observe that both terms are constant except for $\nu_{p} \Psi_{p}^{\prime}$ which will decrease with $D$ as the poor become better off 13 Consequently, when the insurance term is positive for $D=0$, we may get an interior solution at some level $D^{*}$ for which 42 . vanishes or full insurance with $D^{*}=L$. Clearly both the desirability of social insurance and its level depend on $\pi_{r}-\pi_{p}$. When this term is small we can expect a sizeable level of social insurance whereas little or no social insurance will be optimal as the difference in risks increases.

Proposition 3 Assume that some of the high-risk individuals are overconfident concerning their health risk while there continues to be adverse selection in the private insurance market and

\footnotetext{
${ }^{13}$ Recall that we know from $\sqrt{31}$ that they will never be fully insured except when $D=L$.
} 
productivity and risk are positively correlated, then:

(i) Uniform social insurance continues to have a positive insurance and a negative incentive effect. The incentive effect is the same as in the absence of overconfidence, while the insurance term has a different structure.

(ii) Overconfident individuals have no direct relevance when it comes to the desirability of uniform social insurance. Any attempt to redistribute towards this group would be undone by the private market incentive constraints. However, overconfidence comes in indirectly, though because it increases the cost of private insurance for the low-productivity individuals.

\section{Conclusion}

This paper has revisited the role of social insurance to supplement a general income tax. We have assumed that neither public nor private insurers can observe risk. Instead, the private insurance market suffers from asymmetric information, a Rothschild and Stiglitz equilibrium emerges and low-risk individuals are only partly insured. We have concentrated on the case of positive correlation between loss probability and productivity which is relevant for the old-age dependency risk. This is the interesting case from our perspective, because it implies that social insurance, whether uniform or not, is not desirable when private insurance markets are fair.

We have shown that with adverse selection in the insurance market social insurance does have a role to play. When there is no concern for redistribution it can achieve a first-best outcome by completing the market insurance coverage provided to the poor. In the general case, when redistribution is accounted for by adopting a strictly concave welfare function, the solution is second-best. Extending benefits to the poor (or a universal grant) does have adverse incentive effects, but it also corrects a market failure and enhances insurance coverage of the previously underinsured. Uniform coverage was shown to be desirable only when the insurance benefits outweigh the incentive cost. A properly designed non-uniform insurance schedule, on the other hand, is always desirable. Insurance benefits need to be targeted to one of the types only, and quite surprisingly this may be the productive individuals.

Finally, we have examined how the desirability of social insurance and its design are affected by overconfidence concerning the health risk of high-productivity agents. We have shown that overconfidence does not affect the incentive term in the expression but makes the insurance term more complex. Interestingly, the existence of overconfident individuals is of no direct relevance when it comes to the desirability of uniform social insurance. Overconfidence comes in indirectly 
though, because it increases the cost of private insurance for the low-productivity individuals which enhances the benefits they receive from social insurance.

\section{References}

[1] Atkinson, A.B. and J.E. Stiglitz, 1976, "The design of tax structure: direct versus indirect taxation," Journal of Public Economics, 6 (1), 55-75.

[2] Boadway, R., M. Leite-Monteiro, M. Marchand, and P. Pestieau, "Social insurance and redistribution with moral hazard and adverse selection," Scandinavian Journal of Economics, 2006, 108 (2), 279-298.

[3] Brown, J.R. and A. Finkelstein, "The private market for long-term care insurance in the united states: a review of the evidence," Journal of Risk and Insurance, 2009, 76, 5-29.

[4] Chetty, R. and E. Saez, "Optimal taxation and social insurance with endogenous private insurance," American Economic Journal: Economic Policy, 2010, 2 (2), 85-116.

[5] Cremer, H., "Multi-dimensional heterogeneity and the design of tax policies," Baltic Journal of Economics, 2003, 35-46.

[6] Cremer, H. and P. Pestieau, "Redistributive taxation and social insurance," International Tax and Public Finance, 1996, 3, 281-295.

[7] Cremer, H. and P. Pestieau, "Myopia, redistribution and pensions," European Economic Review, 2011, 55 (2), 165-175.

[8] Cremer, H., P. Pestieau and J.-Ch. Rochet, "Capital income taxation when inherited wealth is not observable," Journal of Public Economics, 2003, 87, 2475-2490.

[9] Cremer, H., P. Pestieau and J.-Ch. Rochet, "Direct versus indirect taxation. The design of the tax structure revisited," International Economic Review, 2001, 42, 781-799.

[10] Cremer, H. and K. Roeder, "Long-term care policy, myopia and redistribution," Journal of Public Economics, 2013, 108, 33-43.

[11] Cremer, H., F. Gahvari and N. Ladoux, "Externalities and optimal taxation," Journal of Public Economics, 1998, 70 (3), 343-364. 
[12] Cremer, H. and F. Gahvari, "In-kind transfers, self-selection and optimal tax policy," European Economics Review, 1997, 41 (1), 97-114.

[13] Cristia, J.P., "Rising mortality and life expectancy differentials by lifetime earnings in the united states," Journal of Health Economics, 2009, 28, 984-995.

[14] Gerdtham, U.-G. and M. Johannesson, "Income-related inequality in life-years and quality-adjusted life-years," Journal of Health Economics, 2000, 19 (6), 1007-1026.

[15] Kaplow, L., "On the undesirability of commodity taxation even when income taxation is not optimal," Journal of Public Economics, 2006, 90, 1235-1250.

[16] Laroque, G., "Indirect taxation is superfluous under separability and taste homgeneity," Economics Letters, 2005, 87, 141-144.

[17] Mossin, J., "Aspects of rational insurance purchasing," Journal of Political Economy, 1968, $76(4), 553-568$.

[18] National Academy on an Aging Society, "Alzheimer's disease and dementia: a growing challenge," Challenges of the 21st Century: Chronic and Disabling Conditions, 2000, 11.

[19] Nishimura, Y., "Redistributive taxation and social insurance under adverse selection in the insurance market," International Tax and Finance, 2009, 16, 176-197.

[20] Reuser, M., F.J. Willekens, and L. Bonneux, "Higher education delays and shortens cognitive impairment. A multistate life table analysis of the US health and retirement study," European Journal of Epidemiology, 2011, 26, 395-403.

[21] Rochet, J.-C., "Incentives, redistribution and social insurance," Geneva Papers on Risk and Insurance Theory, 1991, 16 (2), 143-165.

[22] Sandroni, A. and F. Squintani, "Overconfidence, insurance, and paternalism," The American Economic Review, 2007, 97 (5), 1994-2004.

[23] Viscusi, W.K., "Mortality effects of regulatory costs and policy evaluation criteria," RAND Journal of Economics, 1994, 25, 94-109.

[24] Yaari, M.E., "The dual theory of choice under risk," Econometrica, 1987, 55, 95-115. 
[25] Zhou-Richter, T., M. Browne and H. Gründl, "Don't they care? Or, are they just unaware? Risk perceptions and the demand for long-term care insurance," Journal of Risk and Insurance, 2010, 77 (4), 715-747. 